\title{
Biallelic TANGO1 mutations cause a novel syndromal disease due to hampered cellular collagen secretion
}

\section{Caroline Lekszas ${ }^{1 \ddagger}$, Ombretta Foresti ${ }^{2 \ddagger}$, Ishier Raote ${ }^{2 \ddagger}$, Daniel Liedtke Eva-Maria König ${ }^{1}$, Indrajit Nanda ${ }^{1}$, Barbara Vona, ${ }^{1,3}$, Peter De Coster ${ }^{4}$, Rita Cauwels ${ }^{4}$, Vivek Malhotra ${ }^{2 \dagger *}$, Thomas Haaf ${ }^{1+*}$}

${ }^{1}$ Institute of Human Genetics, Julius Maximilians University Würzburg, Würzburg, Germany; ${ }^{2}$ Centre for Genomic Regulation, The Barcelona Institute of Science and Technology, Barcelona, Spain; ${ }^{3}$ Department of Otorhinolaryngology, Head and Neck Surgery, Tübingen Hearing Research Centre (THRC), Eberhard Karls University Tübingen, Tübingen, Germany; ${ }^{4}$ Department of Pediatric Dentistry and Special Care, PaeCoMeDis Research Group, Ghent University Hospital, Ghent, Belgium
*For correspondence: vivek.malhotra@crg.eu(VM); thomas.haaf@uni-wuerzburg.de (TH)

${ }^{\dagger}$ These authors contributed equally to this work

FThese authors also contributed equally to this work

Competing interest: See page 14

Funding: See page 14

Received: 25 August 2019

Accepted: 24 February 2020

Published: 26 February 2020

Reviewing editor: Reinhard Fässler, Max Planck Institute of Biochemistry, Germany

(c) Copyright Lekszas et al. This article is distributed under the terms of the Creative Commons Attribution License, which permits unrestricted use and redistribution provided that the original author and source are credited.

\begin{abstract}
The transport and Golgi organization 1 (TANGO1) proteins play pivotal roles in the secretory pathway. Full length TANGO1 is a transmembrane protein localised at endoplasmic reticulum (ER) exit sites, where it binds bulky cargo within the ER lumen and recruits membranes from the ER Golgi intermediate compartment to create an exit route for their export. Here we report the first TANGO1-associated syndrome in humans. A synonymous substitution that results in exon eight skipping in most mRNA molecules, ultimately leading to a truncated TANGO1 protein was identified as disease-causing mutation. The four homozygously affected sons of a consanguineous family display severe dentinogenesis imperfecta, short stature, various skeletal abnormalities, insulin-dependent diabetes mellitus, sensorineural hearing loss, and mild intellectual disability. Functional studies in HeLa and U2OS cells revealed that the corresponding truncated TANGO1 protein is dispersed in the ER and its expression in cells with intact endogenous TANGO1 impairs cellular collagen I secretion.
\end{abstract}

\section{Introduction}

Collagens are the most abundantly secreted molecules in mammals and needed throughout the whole body for bone mineralization, skin and tissue assembly. Within the endoplasmic reticulum (ER) lumen, newly synthesized procollagens assemble into rigid, rod-like triple helices that are too large for export by the conventional coat protein complex II (COPII)-coated vesicle size of 60-90 nm (Malhotra and Erlmann, 2015; Miller and Schekman, 2013). Over the past years, the ER exit site (ERES) located, transport and Golgi organization one protein (TANGO1) has been identified as a player in the export of bulky cargoes like the collagens (Bard et al., 2006; Malhotra and Erlmann, 2011; Malhotra and Erlmann, 2015; Raote and Malhotra, 2019; Raote et al., 2018; Raote et al., 2017; Saito et al., 2009; Santos et al., 2016; Wilson et al., 2011). TANGO1 is conserved throughout most metazoans and ubiquitously expressed in humans. It comprises 8,142 bp located at chromosome 1q41 and encodes two distinct isoforms, full length TANGO1 and TANGO1-short. Full length TANGO1 consists of 1907 amino acids (aa) and contains an N-terminal signal sequence followed by a Src-homology 3 (SH3)-like domain and a coiled-coil domain in the lumenal portion, as well as two additional coiled-coil domains (CC1 and CC2) and a proline-rich domain (PRD) in the cytoplasmic portion (Figure 1A). TANGO1-short is composed of 785 aa and lacks the lumenal portion contained in TANGO1 (Saito et al., 2009). Together with cTAGE5 encoded by the TANGO1- 
A

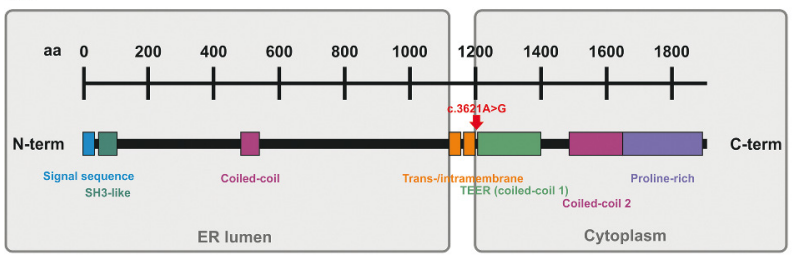

\section{C}

II.2

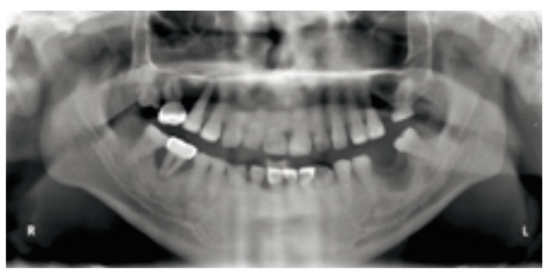

II.4

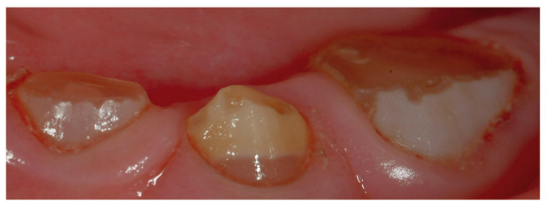

II.5

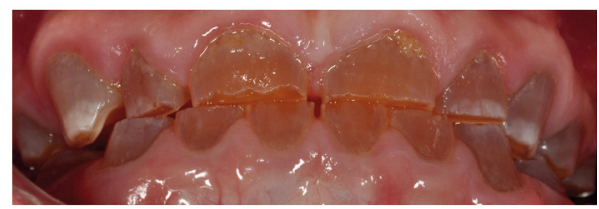

II.2

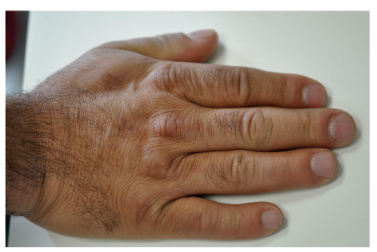

II.4

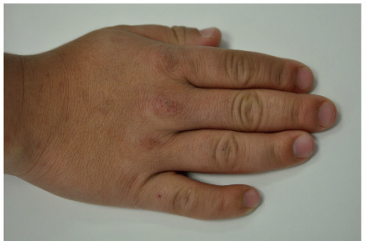

B

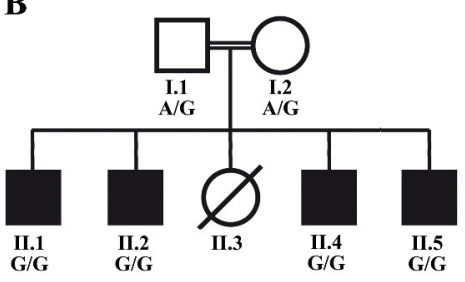


Figure 1 continued

obliteration of the pulp chamber and canals in the permanent dentition), the skin lesions due to pruritus in all affected children; and the scoliosis in II.4 and II.5.

like protein gene (TALI), TANGO1 and TANGO1-short form stable complexes at ERES, to jointly fulfill their roles in the secretion of bulky cargoes such as procollagens, pre-chylomicrons, and large pre-very low-density-lipoproteins (Bosserhoff et al., 2003; Maeda et al., 2016; Malhotra and Erlmann, 2011; Malhotra and Erlmann, 2015; Saito et al., 2009; Saito et al., 2011; Santos et al., 2016; Wilson et al., 2011).

At ERES TANGO1 assembles into rings that enclose COPII coats and create a sub-compartment dedicated to sorting, packing and exporting collagens (Raote and Malhotra, 2019; Raote et al., 2018; Raote et al., 2017). TANGO1's SH3-like domain binds collagens via the collagen-specific chaperone HSP47 (heat shock protein 47) in the ER lumen (Ishikawa et al., 2016). This binding of TANGO1 to HSP47-Collagen is proposed to trigger binding of its PRD to Sec23 in the cytoplasm. TANGO1's CC1 domain, that contains a subdomain named TEER (tether of ER Golgi intermediate compartment at ER), recruits ERGIC-53 membranes, which fuse with the nascent vesicle bud initiated by COPII inner coats ( $\mathrm{Sec} 23 / \mathrm{Sec} 24)$ to grow the collagen filled container into an export conduit (Raote and Malhotra, 2019; Raote et al., 2018; Santos et al., 2015). Subsequent to collagen packing into this conduit, TANGO1 dissociates from HSP47 and collagen. TANGO1 is retained at ERES while collagens move forward in the anterograde direction (Raote and Malhotra, 2019).

The discovery of TANGO1 has made the process by which cells organize ERES and export collagen amenable to molecular analysis. We now describe the first human TANGO1 mutation associated with a novel autosomal-recessive syndrome. These findings underscore the importance of TANGO1 in human (patho)physiology.

\section{Results}

\section{Clinical description}

Four brothers with a similar combination of congenital anomalies two of whom have already been described by Cauwels et al. (2005) were referred for oral examination to the Centre for Special Care, Ghent University Hospital, at the ages of 7 (Figure 1B; II.1;*1988), 3 (II.2;*1990), 6 (II.4;*2006), and $4\left(\right.$ II. $\left.5 ;{ }^{*} 2008\right)$ years, and were followed up until present. Their parents are of Turkish origin and first cousins. The sister (II.3) as well as both parents (I.1 and I.2) were phenotypically normal. All four brothers presented with severe dentinogenesis imperfecta in both primary and permanent dentitions, delayed eruption of the permanent teeth, growth retardation, proportionate short stature, clinodactyly of the fifth finger, brachydactyly, platyspondyly, primary obesity, insulin-dependent diabetes mellitus (<1 IU of insulin/kg/day), sensorineural hearing loss, and mild intellectual disability (ID). Additional facultative symptoms included scoliosis, retrognathia, mild retinopathy, osteopenia, early onset periodontitis with premature tooth loss, hydronephrosis, and microalbuminuria (Figure 1C; Table 1).

\section{Whole exome sequencing (WES) revealed the disease-causing mutation in TANGO1}

WES was performed in the four affected bothers and their parents. After filtering, 10 variants were found to be homozygous in all affected children and heterozygous in both parents (Supplementary file 1). In-depth data analysis revealed a synonymous variant in exon 8 of TANGO1 (NM_001324062.1: c.3621A > G) as the most likely disease-causing mutation. TANGO1 is known to be crucial for the secretion of collagens, consistent with phenotypes in our patients. WES results were validated by Sanger sequencing (Figure 2A). This TANGO1 mutation was not present in large population databases such as ExAC or gnomAD (Lek et al., 2016). Although it does not alter the amino acid at the respective position (p.(Arg1207=)), the A > G substitution was predicted by ESEfinder to disrupt an exon splice enhancer (ESE) motif recognized by the human SR protein SC35 (Figure 2-figure supplement 1A). The mutation affects residue 1207 between the intramembrane and the CC1 domain within the cytoplasmic portion of TANGO1 (Figure 1A). 
Table 1. Clinical symptoms in four affected brothers

\begin{tabular}{|c|c|c|c|c|}
\hline & II.1 & II.2 & II.4 & II.5 \\
\hline Dentinogenesis imperfecta & $x$ & $x$ & $x$ & $x$ \\
\hline Delayed eruption of permanent teeth & $x$ & $x$ & $x$ & $x$ \\
\hline Juvenile periodontitis with early tooth loss & $x$ & $x$ & & \\
\hline Growth retardation & $x$ & $x$ & $x$ & $x$ \\
\hline Proportionate short stature & $x$ & $x$ & $x$ & $x$ \\
\hline High nasal bridge & $x$ & $x$ & $x$ & $x$ \\
\hline Retrognathia & & & & $x$ \\
\hline Phalangeal brachydactyly of fingers & $x$ & $x$ & $x$ & $x$ \\
\hline Clinodactyly of 5th finger & $x$ & $x$ & $x$ & $x$ \\
\hline Cone-shaped epiphyses in the hands & $x$ & $x$ & & $x$ \\
\hline Brachydactyly of toes & & & $x$ & $x$ \\
\hline Platyspondyly (flattened vertebral corpora) & $x$ & $x$ & $x$ & $x$ \\
\hline Scoliosis & & & $x$ & $x$ \\
\hline Prominent knees & $x$ & $x$ & $x$ & $x$ \\
\hline Mild intellectual disability & $x$ & $x$ & $x$ & $x$ \\
\hline Sensorineural hearing loss & $x$ & $x$ & $x$ & $x$ \\
\hline Mild retinopathy & $x$ & & & $\mathrm{x}$ \\
\hline Insulin-dependent diabetes mellitus & $x$ & $x$ & $\mathrm{x}$ & $x$ \\
\hline Primary obesity & $x$ & $x$ & $x$ & $x$ \\
\hline Early onset puberty & & & & $x$ \\
\hline Pruritus & $x$ & $x$ & $x$ & $x$ \\
\hline Asthma & $x$ & $x$ & $x$ & $x$ \\
\hline Osteopenia & $x$ & $x$ & & \\
\hline Hydronephrosis (junctional stenosis) & & & $x$ & \\
\hline Nephropathy (microalbuminuria) & & $x$ & & \\
\hline
\end{tabular}

Homozygosity mapping of two affected brothers (II.1 and II.2) identified a shared homozygous interval of $\sim 19 \mathrm{Mb}$ on chromosome 1, spanning GRCh37/hg19 coordinates 214,413,099233,429,284 (rs12736101-rs6656327), including TANGO1 and 28 disease-associated OMIM genes (Figure 2-figure supplement 1B,C). Apart from TANGO1, none of the shared homozygous intervals was endowed with a pathogenic mutation. In addition, no potentially disease-causing copy number variation (CNV) was detected by array comparative genomic hybridization (CGH).

\section{The TANGO1 mutation leads to exon eight skipping by disrupting an exon splice enhancer both in vivo and in vitro}

In order to investigate possible effects of the identified TANGO1 mutation on pre-mRNA splicing, blood samples of the whole family were used for RNA isolation and reverse transcription PCR. Subsequent gel electrophoresis and cDNA sequencing revealed two splice products in the homozygous sons and their heterozygous parents, one representing the full length transcript being more abundant in the parents and another one lacking the entire exon eight being more abundant in the affected sons (Figure 2B,C; Figure 3). Exon eight skipping during TANGO1 pre-mRNA splicing causes a frameshift and a premature stop codon 27 bp downstream of exon 7 (c.3610_3631delinsTCACGGAACAGCAAATTTCTGAGAAGTTGA) (Figure 2D). The predicted truncated protein lacks almost the entire cytoplasmic portion including CC1/TEER, CC2, and PRD (Figure 1A).

A minigene assay (Figure 2-figure supplement 2) was performed to confirm that the identified TANGO1 mutation is sufficient to induce exon eight skipping. Transfection of cultured HEK293T cells 


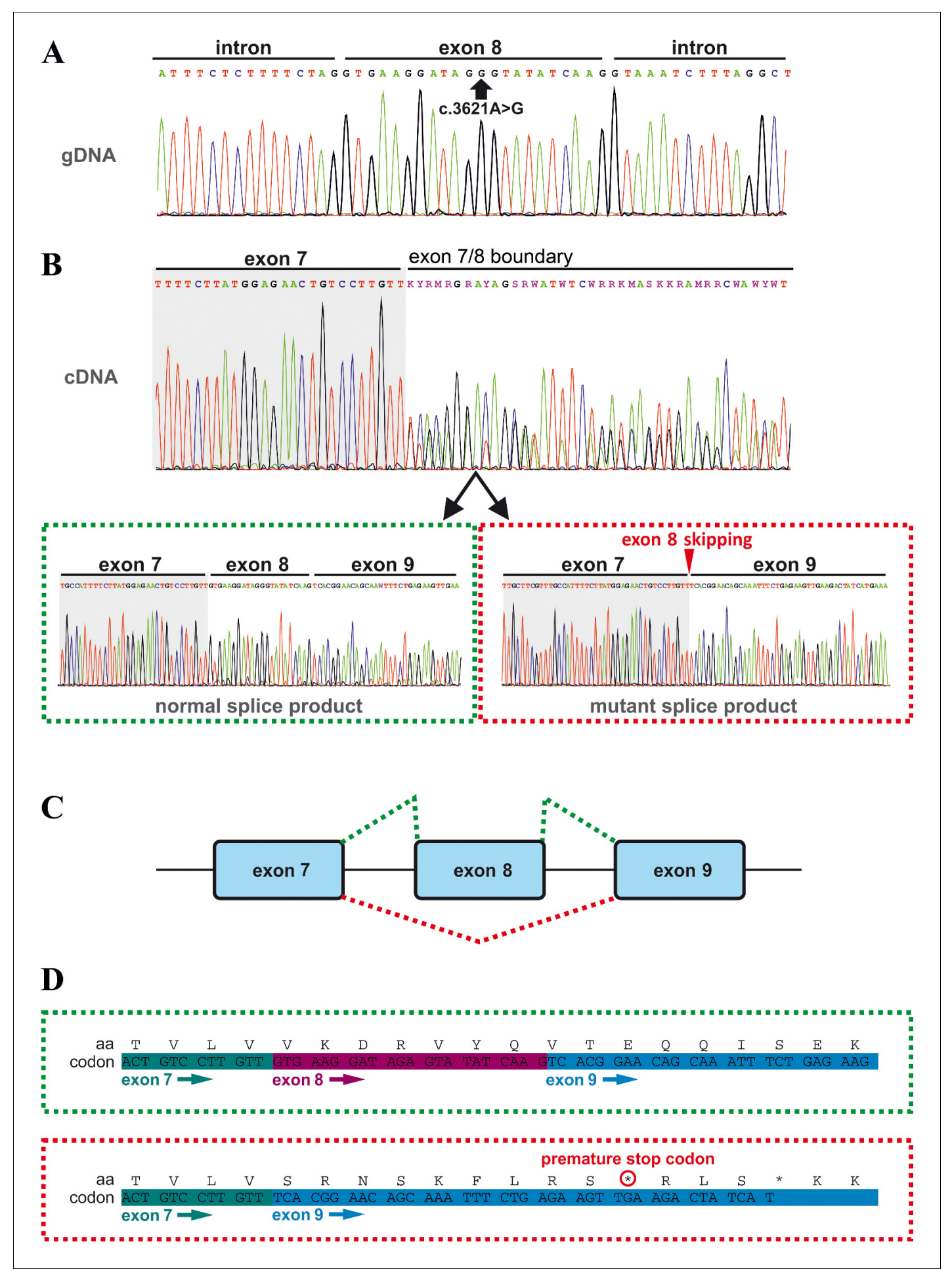

Figure 2. Effects of the TANGO1 (c.3621A > G) mutation on pre-mRNA splicing. (A) The synonymous variant, which was identified by WES and validated by Sanger sequencing in all family members, resides in exon 8 of TANGO1 at genomic position 222,822,182 (GRCh37/hg19). It is predicted to disrupt an exon splice enhancer (ESE) motif recognized by the human SR protein SC35. (B) Electropherograms of the TANGO1 cDNA sequence of one affected child. Note the splitting of the sequence starting at the exon $7 / 8$ boundary. Sequencing of individual bands after gel electrophoretic separation revealed TANGO1 wild-type cDNA and cDNA lacking exon 8 (c.3610_3631delins30). For the cDNA sequencing of TANGO1 splice products, at least two (up to 6) technical replicates were performed for each family member. (C) Schematic representation of the alternatively used splice sites resulting in the normal TANGO1 mRNA (green dotted lines) and in exon eight skipping (red dotted lines). (D) Figure 2 continued on next page 
Figure 2 continued

Consequences of TANGO1 exon eight skipping on the reading frame and the amino acid level. Exclusion of exon eight causes a premature stop codon.

The online version of this article includes the following figure supplement(s) for figure 2:

Figure supplement 1. Sequence analysis.

Figure supplement 2. Minigene assay.

Figure supplement 3. Possible mechanisms underlying TANGO1 exon eight skipping.

with the Mnt vector carrying the mutated (c.3621A > G) TANGO1 exon 8 and 250 bp flanking intronic sequences mainly produced vector-derived splice products lacking exon 8.

The observed splicing error could be due to disruption of an ESE motif recognized by the SR protein SC35 or to formation of a splice repressor motif recruiting the heterogeneous nuclear ribonucleoprotein A1 (hnRNP A1). To test this, cultured HeLa cells were transfected with either a wild-type or a mutated TANGO1 vector (Figure 2-figure supplement 3 ) and then treated with a customized antisense oligonucleotide (vivo morpholino) targeting the entire TANGO1 exon 8. SR proteins are required for proper exon inclusion during splicing and their absence can lead to exon skipping. The observed effects of the morpholino treatment on TANGO1 splicing support the idea that the c.3621A > G mutation interferes with SR protein binding.

\section{The homozygous TANGO1 mutation results in exon eight skipping in most splice products}

Quantitative real-time (qRT) PCR on blood cDNA samples was performed to quantify the amount of mutant and normal TANGO1 splice products, respectively, in homozygous and heterozygous mutation carriers compared to a normal control (Figure 3). The affected children consistently displayed the lowest amounts of normal splice product (mean $R Q$ value: 0.39 ) and the highest amounts of the exon eight skipped product (mean RQ value: 4.58). Both parents showed more normal splice product (mean $\mathrm{RQ}$ value: 0.59 ) than their children but still only approximately half of that of the control. In contrast to the control individual, both parents also displayed a considerable proportion of the exon eight skipped splice product (mean RQ value: 2.66). Because of a processed transcript (ENST00000495210.1) without exon eight which is probably co-amplified by reverse transcription $\mathrm{PCR}$, the exact ratios of the mutant versus the normal splice product could not be determined.

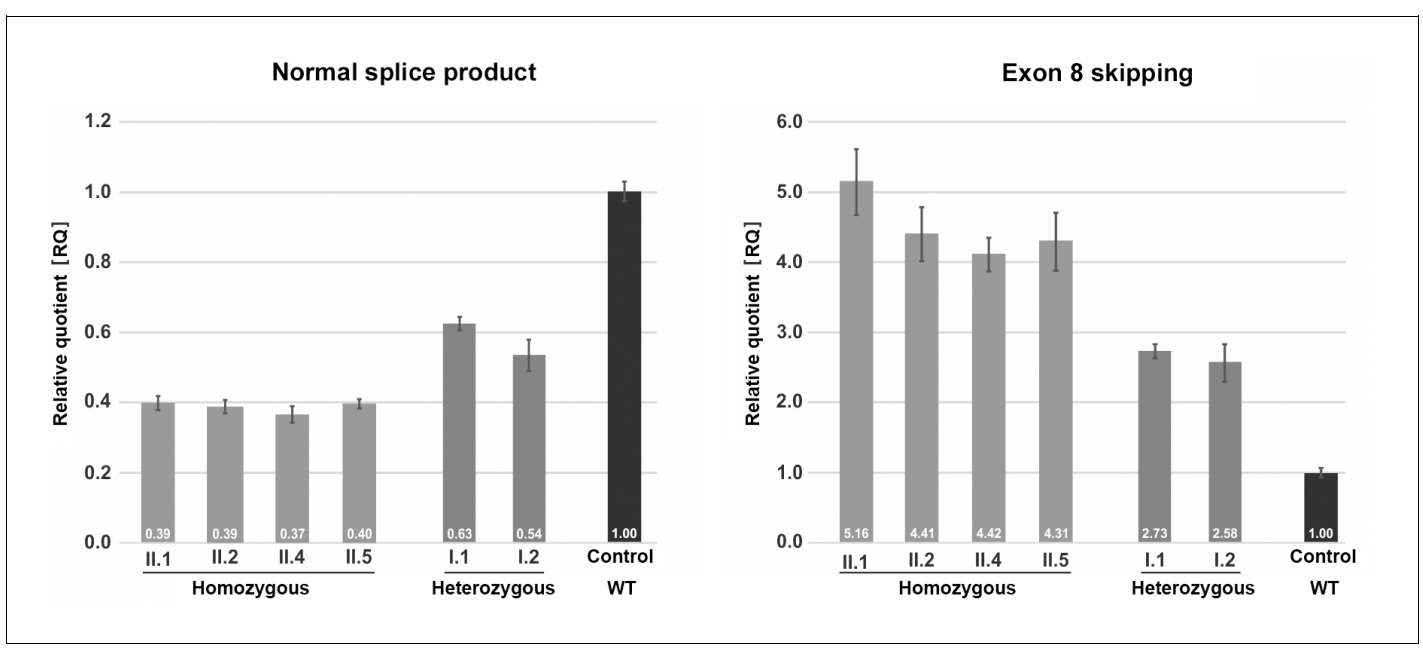

Figure 3. Quantification of TANGO1 splice products in homozygous and heterozygous mutation carriers, compared to a control individual (without mutation). The right bar diagram shows the relative amounts of the normally spliced TANGO1 cDNA and the left diagram of splice products lacking exon 8. The standard deviation of each bar represents the results of triplicate measurements. A control cDNA sample was used for normalisation and relative comparison $(R Q=1)$. By $q R T-P C R$ the control sample used was representative for three other control individuals. 
Unfortunately, it was not possible to design specific primers for the aberrant exon eight skipped splice product. In a homozygous state, the TANGO1 c.3621A > G mutation leads to exon eight skipping in most splice products, whereas in the heterozygous parents the normal splice product is more abundant.

\section{The truncated TANGO1 protein does not localise to ER exit sites}

To test the properties of the truncated TANGO1 protein, TANGO1 lacking exon 8 (Ex8-HA) was expressed in cultured U2OS cells. Cells were transiently transfected with cDNA for either wild-type

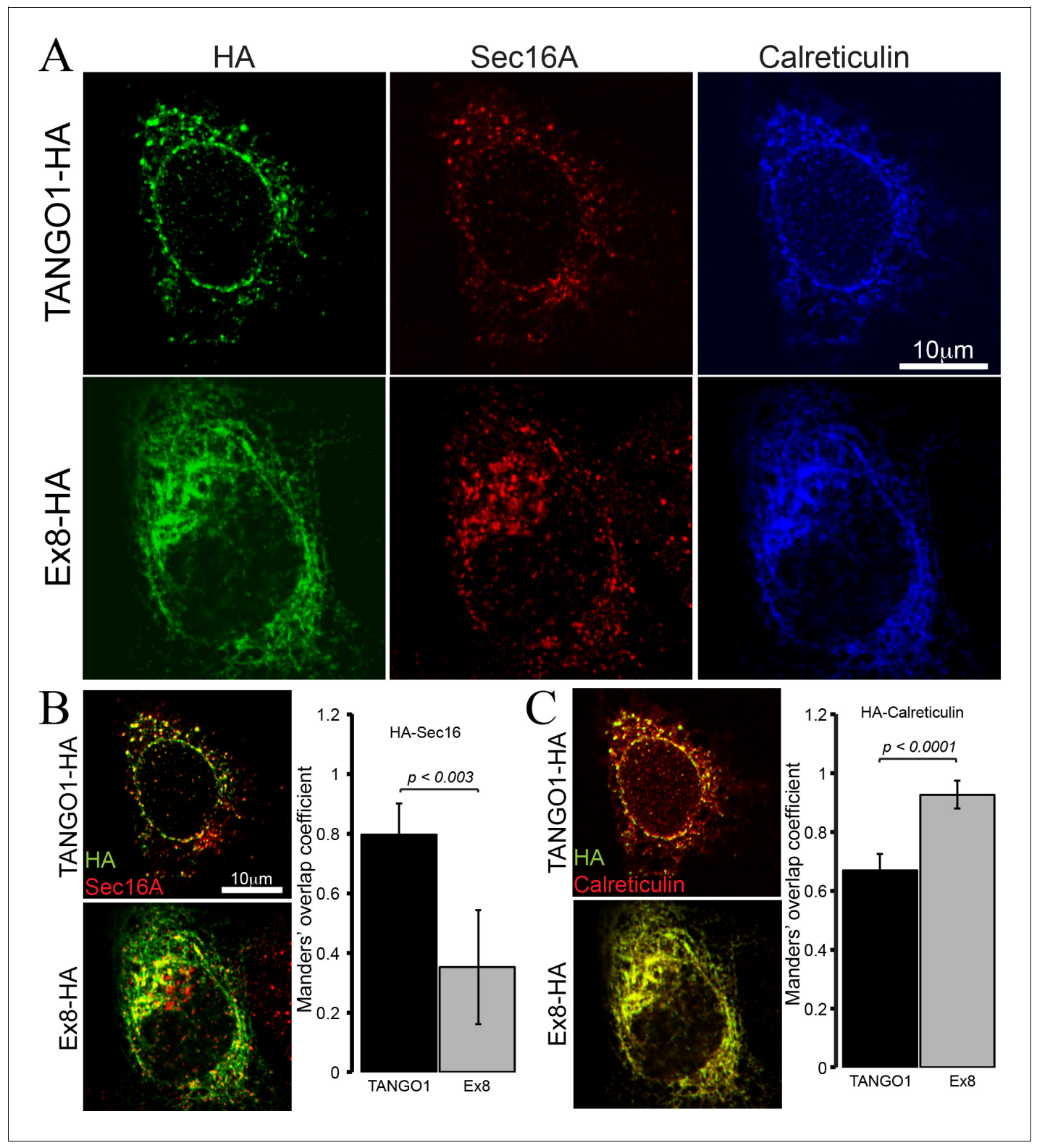

Figure 4. Ex8 mutant does not localise to ER exit sites. Immunofluorescence images of U2OS cells, transiently transfected with WT TANGO1-HA or Ex8-HA. Representative images of three independent experiments. (A) Cells were probed with anti-HA (green), anti-Sec16A (red) and anti-Calreticulin (blue) antibodies. Scale bar $10 \mu \mathrm{m}$. (B) Merged images of TANGO1-HA or Ex8-HA (green) and sec16A (red) and a plot comparing Manders' overlap coefficient of HA with sec16A in TANGO1- or Ex8-expressing U2OS cells. (C) Merged images of TANGO1-HA or Ex8-HA (green) with calreticulin (red) and a plot comparing Manders' overlap coefficient of HA and calreticulin in TANGO1- or Ex8-expressing U2OS cells. Student's t test was performed to compare the Manders' overlap coefficients, $p$ values are shown. 
TANGO1-HA or Ex8-HA (Figure 4). Forty-eight hours after transfection, cells were fixed, permeabilised and immunostained for HA (green), the ERES marker Sec16A (red) and the ER marker calreticulin (blue) (Figure 4A). WT TANGO1-HA (green) expressed in distinct puncta, which colocalised with Sec16A (red). On the other hand, Ex8-HA (green) was distributed in a more diffused pattern throughout the cell and it did not localise to ERES (red) (Figure 4B). WT TANGO1-HA (green) showed less association with calreticulin (red), while Ex8-HA (green) was almost entirely colocalised with calreticulin (red) (Figure 4C). These data are consistent with our understanding of TANGO1 function, as its cytoplasmic domains are required to recruit TANGO1 to ERES. The Ex8 mutant lacks any cytoplasmic domains and consequently is distributed through the ER.

\section{Cells expressing the truncated TANGO1 show reduced levels of intracellular and secreted collagen I}

All affected individuals showed the highest amounts of the exon eight skipped splice product compared to the normal TANGO1 splice product (Figure 3). However, it was not possible to determine how the relative abundance of the two splice products translated to protein levels in patient-derived samples. Therefore, possible effects of the overexpression of Ex8-HA on top of endogenous levels of normal TANGO1 splice product at the cellular level were investigated using human osteosarcoma U2OS as a model system. U2OS cells produce and rapidly secrete collagen I, so they are the ideal system to monitor possible effects of Ex8-HA overproduction on collagen homeostasis. For this purpose, a stable U2OS cell line expressing Ex8-HA under a constitutive promoter was generated and compared with control cells by microscopy. Cells expressing Ex8-HA showed weaker and more diffuse staining of collagen I compared with control cells (Figure 5-figure supplement 1A). To confirm that this reduction was not due to unequal immunofluorescent staining, total RNA was isolated from the two cell populations and the relative amount of collagen I/GAPDH transcript was quantified by qRT-PCR analyses (Figure 5-figure supplement 1B). This confirmed that collagen I expression is reduced in the Ex8-HA stable cell line. The reduction in expression was not due to unfolded protein response (UPR) as there was no difference in XBP-1 splicing upon expression of Ex8-HA (Figure 5-figure supplement $1 \mathrm{C}$ ).

The next step was to test whether the secretion of collagen I was also affected. Since different rates of collagen synthesis will affect the amount available for secretion in the two cell populations, a cycloheximide chase experiment was performed (Figure 5). By inhibiting protein synthesis, it was possible to monitor the rate of secretion of the available pool of collagen I present in the cells at time zero. By quantifying the relative amount of collagen I present in the cells and in the media at each time point, a drastic reduction in the rate of collagen I secretion from the EX8-HA stable cell line compared to control cells was observed. Importantly, this effect was not due to a general reduction of protein secretion since the small cargo antitrypsin was produced and secreted at a comparable rate in the two cell populations. Under the same conditions, we tested secretion of collagen IV and collagen XII in EX8-HA stable line. Six independent trials revealed a consistent trend of a net reduction in their secretion, but because of the low relative abundance of these collagens the magnitude of the effect varied considerably and cannot be statistically quantified (Figure 5-figure supplement 2).These secretion defects were specific to Ex8 expression as we observed no change in collagen I secretion when full-length TANGO1 was overexpressed in U2OS (Figure 5-figure supplement 3). Collectively, these results show that expression of the exon eight skipped splice product even in the presence of full length TANGO1 affects collagen I homeostasis.

\section{Discussion}

Our study provides evidence that aberrant expression of a truncated TANGO1 protein and/or reduced levels of fully functional TANGO1 protein, or likely a combination of both causes a novel syndrome due to disturbances in cellular protein secretion. The heterozygous parents show exon eight skipping but no detectable symptoms, whereas all four homozygous children are severely and similarly affected. This is consistent with a threshold model, where the disease only manifests when the ratio of truncated versus normal protein exceeds a critical level. Tango1 knockout mice represent a full loss-of-function (LoF) situation and are defective for the secretion of numerous collagens, exhibiting short-limbed dwarfism, compromised chondrocyte maturation and bone mineralization, and other features (Wilson et al., 2011), resembling our patients' phenotype. The human TANGO1 


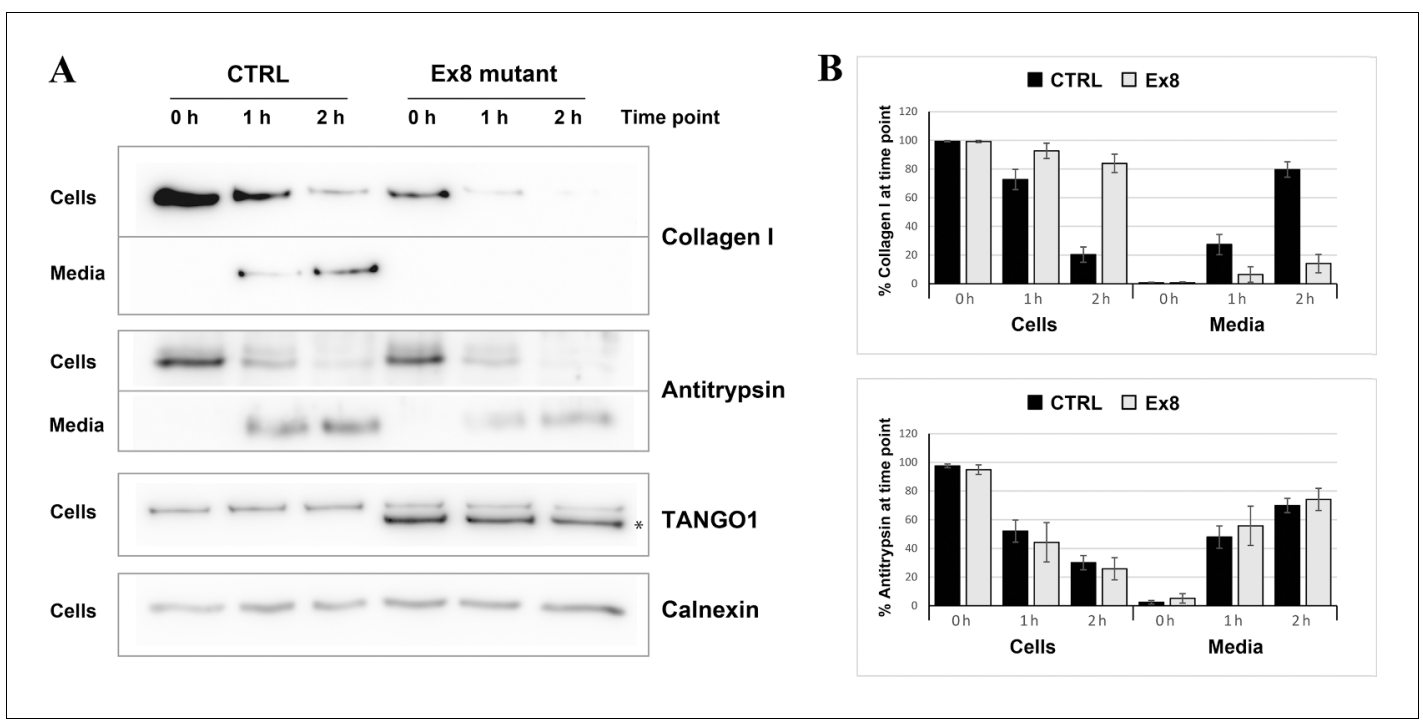

Figure 5. Ex8 mutant expression reduces collagen I secretion in U2OS cells. (A) Media of U2OS control cells (CTRL) or stably expressing Ex8-HA mutant (Ex8) were replaced with OptiMEM media containing $0.25 \mathrm{mM}$ ascorbic acid and $50 \mu \mathrm{M}$ cycloheximide to block protein synthesis and follow collagen secretion. Cell extracts and media were collected at the indicated time points and analysed by SDS-PAGE followed by Western blotting with antibodies raised against Collagen I, TANGO1, and Calnexin (loading control). (*) indicates Ex8-HA. Representative images of four independent experiments. (B) For each time point, the band intensities of collagen I (upper panel) or antitrypsin (lower panel) were measured for the cell extract and media samples and expressed as percentage of the total (cells plus media). Each graph represents the average quantification of four experiments and corresponding standard deviations.

The online version of this article includes the following figure supplement(s) for figure 5 :

Figure supplement 1. TANGO1 exon eight mutant (Ex8) expression reduces collagen I expression in U2OS cells without inducing UPR.

Figure supplement 2. Ex8 mutant expression reduces collagen XII and collagen IV secretion in U2OS cells. Figure supplement 3. TANGO1 full-length overexpression has no effect on collagen I secretion in U2OS cells.

locus lies within a homozygous interval shared among the affected children, which strengthens its role as the disease-causing gene in the investigated family. TANGO1 does not seem to be haploinsufficient, since there are several heterozygous LoF mutation carriers listed in big population databases, ExAC and gnomAD (Lek et al., 2016). However, no homozygous LoF mutation carriers have been reported so far. Thus, complete ablation of functional TANGO1 may cause embryonic lethality in humans.

The skipped exon eight in full length TANGO1 corresponds to exon three in the isoform TANGO1-short. Its exclusion there also leads to a premature stop codon 27 bp downstream of exon 2. TANGO1-short has a similar structure to TANGO1, but lacks the lumenal portion within the cargo-binding SH3 domain. However, TANGO1-short has been shown to substitute the function of TANGO1 in collagen export, and vice versa (Maeda et al., 2016). It has been postulated that the cytoplasmic portion's capacity to recruit ERGIC-53 membranes, Sec23/24 complexes, and cTAGE5, shared by both isoforms, is sufficient to export collagen at the ER (Saito et al., 2009; Santos et al., 2015). The lumenal SH3 domain may therefore rather play a role in modulating the efficiency or the quality (folding status) of collagens to be secreted (Maeda et al., 2016; Raote et al., 2018; Saito et al., 2009). Since the identified mutation in exon eight does not only compromise the function of TANGO1, but most likely also of TANGO1-short, the short isoform cannot attenuate our patients' phenotype.

The truncated TANGO1 protein is unable to access ERES and is distributed throughout the ER (Figure 4). Its expression in cells along with endogenous full length TANGO1 displayed a negative effect on collagen secretion. This effect is specific to collagen, as the secretion of antitrypsin remains unaffected (Figure 5). In two previous descriptions of TANGO1 function (Nogueira et al., 2014; Saito et al., 2009), we reported that it had no obvious role in collagen I export. However, it has 
been demonstrated subsequently that TANGO1 functions to export all soluble collagens tested thus far, including collagen I. We have noticed a change in the mRNA level of collagen $\alpha 1$ chain in U2OS cells expressing mutant TANGO1. Although, we have not tested the effects on the expression of other chains of collagen one and other collagens, it is conceivable that expression of this mutant causes accumulation of collagens in the ER, which then affect their further synthesis by a feedback mechanism. However, this appears not to involve the activation of UPR in U2OS cells. Whether this is also the situation in vivo in the individuals with TANGO1 mutation is unknown.

Type I collagens provide tensile strength to connective tissue and are abundant in bone, skin, dentin, cementum, tendons, and ligaments (Deshmukh et al., 2016). Impaired collagen I secretion may underlie several key symptoms in our patients, in particular the tooth and skeletal abnormalities. Haploinsufficiency or gain of function mutations in COL1A1 and COL1A2, coding for the pro-collagen I chains, can cause different forms of osteogenesis imperfecta (OI) with (OMIM \#259420) or without dentinogenesis imperfecta (DGI). Hearing loss is commonly found in OI patients. Mutations in other collagen genes, for example COL2A1, COL4A3, COL4A4, COL4A5, COL4A6, COL9A1, COL9A2 and COL9A3, COL11A1, and/or COL11A2 have been associated with hereditary hearing loss (https://hereditaryhearingloss.org/). TANGO1 may control secretion of cargoes other than collagens. This is particularly noteworthy based on the recently reported role of TANGO1 in mucin export in Drosophila salivary glands and insulin secretion (Fan et al., 2017; Kang et al., 2019; Reynolds et al., 2019) Therefore, the complex phenotype of our patients, including DGI, diabetes, intellectual disability and hearing impairment, but no brittle bones may at least partially be due to aberrant export and secretion of other proteins in a tissue specific manner.

For example, mutations in the dentin sialophosphoprotein (DSPP) gene and, by extrapolation, TANGO1-associated defects in DSPP secretion, may cause DGI 1 (\#605594) with or without hearing loss (Xiao et al., 2001) as well as DGI, Shields type II (\#125490) and III (\#125500), which may be phenotypic variation of the same entity rather than separate diseases (Kim et al., 2005).

Defective secretion of other molecules may cause diabetes mellitus and pubertas praecox. Endocrinological examination revealed that the glucose intolerance of the affected children is due to reduced levels of secreted insulin. cTAGE5 is known to cooperate with TANGO1 in the mega cargo secretion pathway (Saito et al., 2011), but has also been shown to play a pivotal role in ER to Golgi trafficking of small molecules like proinsulin (Fan et al., 2017). The knockout of cTAGE5 in pancreatic $\beta$-cells resulted in defective islet structure, reduced insulin secretion, and severe glucose intolerance in mice (Fan et al., 2017). Additionally, a correlation between TANGO1 phosphorylation and proinsulin trafficking in mouse pancreatic $\beta$-cells has recently been discovered (Kang et al., 2019). In this light, it will be intriguing to investigate the role of TANGO1 in the insulin secretion pathway in future studies.

Collectively, the investigated family presents the first TANGO1-associated syndrome in humans, highlighting the role of fully functional TANGO1 in various disease pathways and bringing new potential target molecules of TANGO1 into focus.

\section{Materials and methods}

\section{Whole exome sequencing}

Exome capture was performed according to the Illumina Nextera Rapid Capture Enrichment library preparation (individuals II.1 and II.2) or the Illumina TruSeq Rapid Exome library preparation kit (individuals I.1, I.2, II.4, and II.5), using $50 \mathrm{ng}$ of genomic DNA. Paired-end sequencing of the libraries was performed with a NextSeq500 sequencer and the v2 reagent kit (Illumina, San Diego, California, USA). Sequences were mapped to the human genome reference (NCBI build37/hg19 version) using the Burrows-Wheeler Aligner. Aligned reads ranged between 82,649,383 and 102,537,469. The mean coverage was $\geq 52$ with $90.3 \%$ of the exome being covered at least $10 x$. A total of 237,330 297,312 variants per sample were called and analyzed using GensearchNGS software (PhenoSystems SA, Braine le Chateau, Belgium). Variants with a coverage of $\leq 20$, a Phred-scaled quality of $\leq 15$, a frequency of $\leq 20$, and a MAF of $\geq 1 \%$ were neglected. Two control samples from healthy individuals were used for filtering out platform artefacts. Alamut Visual (Interactive Biosoftware, Rouen, France) software including prediction tools like SIFT, MutationTaster, and PolyPhen-2 was used for variant prioritization. Potential effects of a variant on pre-mRNA splicing were evaluated by 
SpliceSiteFinder-like, MaxEntScan, NNSPLICE, GeneSplicer, Human Splicing Finder, ESEfinder, RESCUE-ESE, and EX-SKIP. Population databases like EXAC, gnomAD, and GME revealed whether a variant has been previously found. Protein expression, structure, and functional aspects were investigated with UniProt and The Human Protein Atlas. Information on mouse models was retrieved from the MGI database.

\section{Sanger sequencing}

TANGO1 exon eight was amplified by a touchdown PCR program using primers in the flanking introns (forward 5'-TCAGACCACAACATATCACTACTGG-3'; reverse 5'-TACTCTATCATACAACC TGGCAACC-3'). A clean-up step with ExoSAP-IT (Applied Biosystems, Foster City, California, USA) was followed by the sequencing reaction using the BigDye Terminator Cycle Sequencing Kit v1.1 (Applied Biosystems). Sequencing was conducted on a 3130XL capillary sequencer (Applied Biosystems) and data analysis was performed with Gensearch (PhenoSystems SA).

\section{Microarray analyses}

Two affected children (II.1 and II.2) were genotyped by Life and Brain (Bonn, Germany), using the Infinium Global Screening Array-24 v1.0 BeadChip (Illumina). Shared homozygous intervals were identified with HomozygosityMapper (Seelow et al., 2009).

Array CGH was performed using the CGX DNA labeling kit (PerkinElmer, Waltham, Massachusetts, USA) and the CGX-HD array (PerkinElmer) that covers clinically relevant regions with 180,000 oligonucleotide marker. A male genomic DNA sample served as a reference. The hybridized array was scanned with the NimbleGen MS 200 Microarray Scanner (Roche, Basel, Switzerland). Data analysis was conducted with CytoGenomics 2.5 (Agilent Technologies, Santa Clara, California, USA) and Genoglyphix 3.0 (PerkinElmer) software using annotations from GRCh37/hg19.

\section{Minigene assay}

To investigate possible effects of the TANGO1 mutation on pre-mRNA splicing, the homozygous individual II.1 was compared to a normal control sample in a minigene assay. The plasmid pSPL3bcam vector (Burn et alo, 1995) is endowed with a chloramphenicol resistance, an SV40 promoter, SD6 and SA2 primer sequences, as well as a multiple cloning site including recognition sites for Xhol and BamHI (Figure 2-figure supplement 2A). A 521 bp amplicon including TANGO1 exon eight and $\sim 250$ bp flanking intronic sequences was generated from genomic DNAs of the patient and a control, using primers with recognition sites for Xhol and BamHl at their $5^{\prime}$ ends (fwd $5^{\prime}$-AATTC TCGAGTATCTTTAGCTGTGCAAAGT-3'; rev 5'-ATTGGATCCAAGGTCAATCTGCCCCAAAT-3') and the Q5 High-Fidelity DNA Polymerase (New England Biolabs, Ipswich, Massachusetts, USA). The PCR products were purified with the GenElute PCR Clean-Up kit (Sigma-Aldrich, St. Louis, Missouri, USA), digested with Xhol and BamHl in CutSmart Buffer (New England Biolabs), again purified, and finally ligated into the linearized vector, using T4 DNA Ligase and T4 DNA Ligase Reaction Buffer (New England Biolabs).

Vector constructs were transformed into $\mathrm{DH} 5 \alpha$ bacteria by heat shock for $90 \mathrm{~s}$ at $42^{\circ} \mathrm{C}$ and then plated onto LB/agar/chloramphenicol Petri dishes. Following overnight incubation at $37^{\circ}$, a colony screen was performed using SD6 (fwd 5'-TCTGAGTCACCTGGACAACC- $3^{\prime}$ ) and the TANGO1 exon eight reverse primer (see above). Positive clones (with insert) were cultured overnight and the vector constructs extracted using the GenElute Plasmid Miniprep kit (Sigma-Aldrich). Sequencing was performed with $100 \mathrm{fmol}$ of vector constructs, SD6 forward and TANGO1 exon eight reverse primers. Three vector constructs were selected for splicing experiments, one with the wild-type TANGO1 exon eight and flanking sequences (WT vector) and another one with the mutated TANGO1 exon 8 (Mnt vector). The pSPL3b-cam vector without insert (CTRL) served as control.

Aliquots of $4 \times 10^{15}$ HEK293T cells were plated into 6-well-plates and transfected with vector constructs (WT, Mnt, or CTRL) using the FuGENE HD Transfection Reagent (Roche). After $24 \mathrm{hr}$ of incubation at $37^{\circ} \mathrm{C}$, RNA was isolated with the miRNeasy Mini kit (Qiagen, Venlo, Netherlands). cDNA was synthesized using the High Capacity RNA-to-cDNA kit (Applied Biosystems), amplified by a touchdown PCR program using SD6 forward and SA2 reverse (5'-ATCTCAGTGGTATTTGTGAGC$3^{\prime}$ ) primers, purified with ExoSAP-IT (Applied Biosystems) and finally sequenced. The resulting splice products were compared by gel electrophoresis and cDNA sequencing. Since the Mnt vector 
produced two separate TANGO1 splice products, individual cDNA molecules were cloned using the TA Cloning Kit and the Dual Promoter ( $p C R I I)$ protocol (Invitrogen, Carlsbad, California, USA).

\section{RNA isolation from whole blood samples, cDNA synthesis and sequencing}

Peripheral blood samples were collected in PAXgene Blood RNA tubes and RNA was isolated using the PAXgene Blood RNA Kit (PreAnalytiX, Hombrechtikon, Switzerland). Reverse transcription was performed with the High Capacity RNA-to-cDNA Kit (Applied Biosystems). cDNA was amplified by a touchdown PCR program using primers located in TANGO1 exon 6 and 11, respectively (fwd $5^{\prime}$ ACACTCCTATGGATGCTATTGATGC-3'; rev 5'-CTCTCTCAGATTCTAGCATAACACG-3'). After a clean-up step with ExoSAP-IT (Applied Biosystems), cDNA sequencing was performed with the BigDye Terminator Cycle Sequencing Kit v1.1 (Applied Biosystems) and a 3130XL capillary sequencer (Applied Biosystems). Data analysis was performed with Gensearch (PhenoSystems SA) and CodonCode Aligner (CodonCode Corporation, Centerville, Massachusetts, USA). Multiple PCR products were separated by gel electrophoresis. cDNA of individual cut out bands was isolated with the OIAquick Gel Extraction Kit (Qiagen) and 1-3 $\mu$ l of gel extracts were used for sequencing. At least two technical replicates were performed for each analyzed individual.

\section{Morpholino assays}

Effects of different vivo morpholinos on TANGO1 pre-mRNA splicing were tested on HeLa cells with a different genetic background. $2 \times 10^{5}$ HeLa cells in $2 \mathrm{ml}$ DMEM (Sigma-Aldrich) each were plated into the required number of wells of a 6-well-plate and incubated at $37^{\circ} \mathrm{C}$ for $24 \mathrm{hr}$. Depending on the research question, cells were then transfected with the TANGO1 WT or Mnt vector, or used without vector transfection for morpholino treatment. For transfection, $2 \mu \mathrm{g}$ vector were suspended in up to $95 \mu \mathrm{l}$ DMEM and mixed with $6 \mu \mathrm{l}$ FuGENE HD Transfection Reagent (Roche). The mixture was incubated at room temperature for $15 \mathrm{~min}$ and then slowly added to the cells.

The customized TGO morpholino (TACCTTGATATACTCTATCCTTCAC) targets the entire TANGO1 exon 8. A standard control morpholino (GeneTools, Philomath, Oregon, USA) was used to exclude unspecific effects. The vivo morpholinos were added at a final concentration of 5 or $7 \mu \mathrm{M}$. After $24 \mathrm{hr}$ incubation, the medium was removed, cells were washed with $2 \mathrm{ml} 1 \mathrm{x}$ PBS, detached with $0.5 \mathrm{ml}$ Trypsin-EDTA Solution (Sigma-Aldrich) and then transferred into $2 \mathrm{ml}$ tubes with 1x PBS. Samples were centrifuged at $3000 \mathrm{~g}$ for $5 \mathrm{~min}$ at $4^{\circ} \mathrm{C}$. Supernatant was removed and RNA isolated using the miRNeasy Mini Kit (Qiagen). cDNA synthesis and sequencing were performed as described above, using vector-specific primers (SD6 and SA2). This ensured that only vector-derived splice products were analysed.

\section{Quantitative real-time PCR and XBP-1 splicing}

qRT-PCRs were performed to obtain a relative ratio of either the exon eight skipped TANGO1 or the normal splice product for homozygous and heterozygous mutation carriers, compared to a reference sample, which was found to be representative for normal individuals (without mutation). Two housekeeping genes, HPRT1 (fwd 5'-TGACACTGGCAAAACAATGCA-3'; rev 5'-GGTCCTTTTCACCAGCAAGCT-3') and IPO8 (fwd 5'-CGAGCTAGATCTTGCTGGGT-3'; rev 5'-CGCTAA TTCAACGGCATTTCTT-3') served as endogenous controls. Assay 1 (fwd $5^{\prime}$-GACTGCCATGGAAACC TGTATT-3'; rev 5'-TCCGTGAAACAAGGACAGTTCT-3') exclusively amplified the mutant splice product where exon seven is followed by exon 9; assay 2 (fwd 5'-GACTGCCATGGAAACCTGTATT$3^{\prime}$; rev $5^{\prime}$-CCTTCACAACAAGGACAGTTCT-3') the normal splice products where exon seven is followed by exon 8. The PCR reaction consisted of $4 \mu \mathrm{l}(10 \mathrm{ng}) \mathrm{cDNA}, 1 \mu \mathrm{l}(2.5 \mathrm{pmol})$ primer pair, $2 \mu \mathrm{l}$ $5 x$ HOT FIREPol EvaGreen qPCR Mix Plus, and $3 \mu \mathrm{l}$ water. All samples were run in technical triplicates. Cycling conditions on a ViiA 7 Real-Time PCR System (Applied Biosystems) were as follows: $95^{\circ} \mathrm{C}$ for $15 \mathrm{~min}, 40$ cycles of $95^{\circ} \mathrm{C}$ for $15 \mathrm{~s}, 60^{\circ} \mathrm{C}$ for $20 \mathrm{~s}$, and $72^{\circ} \mathrm{C}$ for $20 \mathrm{~s}$. The melt curve was obtained from $60^{\circ} \mathrm{C}$ to $95^{\circ} \mathrm{C}$ and indicated no secondary amplicons.

In addition, qRT-PCRs were conducted to measure the relative expression of full length TANGO1, truncated TANGO1, and Collagen I. For this purpose, U2OS cells WT or stabling expressing Ex8-HA were lysed and total RNA extracted with the RNeasy extraction kit (Qiagen). cDNA from two biological replicates was synthesized with Superscript III (Invitrogen). Primers for COL1A1 (fwd 5'-GTGG 
TCAGGCTGGTGTGATG-3'; rev 5'-CAGGGAGACCCTGGAATCCG-3'), TANGO1 lumenal portion (fwd 5'-TGGAAGTGTTGGACGCACTTTT-3'; rev 5'-TCAGGTTCAGGTTCCCTTTCCT-3') or cytosolic portion (fwd 5'-CTCAGCTCTGCGGACCTTTT3'; rev 5'-GTGAACAGTCCTGGCTAGTGC-3') were designed using Primer-BLAST (NCBI) (Ye et al., 2012) with the annealing temperature to $60^{\circ} \mathrm{C}$. To determine expression levels of collagen I and the two forms of TANGO1, qRT-PCR was performed with Light Cycler 480 SYBR Green I Master (Roche) according to manufacturer's instructions. For each biological replicate, three technical replicates were used to determine mean values and standard deviations.

PCR amplification of XBP-1 from cDNA was done as described previously using 5'-AAACAGAG TAGCAGCTCAGACTGC-3' and 5'-TCCTTCTGGGTAGACCTCTGGGAG-3' (Calfon et al., 2002). IRE1 couples endoplasmic reticulum load to secretory capacity by processing the XBP-1 mRNA. PCR products were separated in $3 \%$ agarose gels. Fragment corresponding to unspliced version of XBP-1 (473 bp) was cut upon Pstl digestion (290 bp and $183 \mathrm{bp}$ ). Fragment corresponding to spliced product was not affected, since the Pstl restriction site is lost after IRE1-mediated cleavage and splicing of the mRNA.

\section{Cell culture and transfection}

HeLa, HEK293T and U2OS cells (obtained from the eukaryotic cell line collection maintained by the Centre for Genomic Regulation, Spain) were grown at $37^{\circ} \mathrm{C}$ with $5 \% \mathrm{CO}_{2}$ in complete DMEM with $10 \%$ FBS. For lentiviral infection of Ex8-HA into U2OS cells, lentiviral particles were produced by cotransfecting HEK293 cells with pHRSIN/Ex8-HA or pHRSIN/TANGO1-HA plasmid and a third-generation packaging vector pool using TransIT-293 (Mirus Bio, Madison, Wisconsin, USA). 72 hr after transfection, the viral supernatant was harvested, filtered, and directly added to U2OS cells. Infected cells were selected using $500 \mu \mathrm{g} / \mathrm{ml}$ hygromycinB (Invitrogen). All cell lines were confirmed to be free of mycoplasma contamination on a monthly basis.

\section{Collagen-secretion assays}

The media of U2OS cells was replaced with OptiMEM medium (Thermo Fisher Scientific, Waltham, Massachusetts, USA) containing $0.25 \mathrm{mM}$ ascorbic acid and $50 \mu \mathrm{M}$ cycloheximide (Sigma-Aldrich) for up to $2 \mathrm{hr}$ to allow for collagen secretion. The media were collected at 0,1 , and $2 \mathrm{hr}$ time points, centrifuged at low speed to remove any cells or cellular debris, and the supernatants were denatured at $65^{\circ} \mathrm{C}$ for $10 \mathrm{~min}$ with Laemmli SDS sample buffer. For cell extracts, cells were washed with PBS, lysed in buffer A (50 mM Tris-Cl, pH7.4, 150 mM NaCl, 1 mM EDTA, 1\% Triton X-100) plus proteases inhibitors (Roche), and centrifuged at $14,000 \mathrm{rpm}$ for $15 \mathrm{~min}$ at $4^{\circ} \mathrm{C}$. The supernatants were denatured at $65^{\circ} \mathrm{C}$ for $10 \mathrm{~min}$ with Laemmli SDS sample buffer. Media and cell lysate were subjected to SDS-PAGE (6\% or $8 \%$ acrylamide) and Western blotting with antibodies raised against collagen I, collagen XII, collagen IV, TANGO1, calnexin, hemagglutinin (HA), and antitrypsin. Band intensities were measured using QuantityOne (Bio-Rad, Hercules, California, USA), and four independent repetitions of the experiment were used to plot the graph. For each time point, the band intensities of collagen I or antitrypsin were measured for the cell extract and media samples, and expressed as percentage of the total (cells plus media). Each graph represents the average quantification of four experiments and corresponding standard deviations.

\section{Immunofluorescence staining}

Cells grown on coverslips were fixed with cold methanol for $10 \mathrm{~min}$ at $-20^{\circ} \mathrm{C}$ and incubated with blocking reagent (Roche) for $30 \mathrm{~min}$ at RT. Primary antibodies were diluted in blocking reagent and incubated overnight at $4^{\circ} \mathrm{C}$. Secondary antibodies conjugated with Alexa Fluor 488, 594 or 647 (Invitrogen) were diluted in blocking reagent and incubated for $1 \mathrm{hr}$ at room temperature. Images were taken with a TCS SP8 or TCS SPE confocal microscope (Leica Microsystems, Wetzlar, Germany) with a $63 \times$ objective. Images processing was performed with ImageJ. Images are representative of three independent experiments. Manders' overlap coefficient was calculated using ImageJ plugin JACoP. Student's t test was performed to compare the Manders' overlap coefficients. 


\section{Antibodies}

Antibodies used in Western blotting and immunofluorescence microscopy were as follows: TANGO1 (Sigma-Aldrich); beta-tubulin (Sigma-Aldrich); calreticulin (Novus Biologicals, Centennial, Colorado, USA); Calnexin (Abcam, Cambridge, United Kingdom); antitrypsin Ab-1 (NeoMarkers, Fremont, California, USA); collagen I and collagen IV (Abcam, Cambridge, United Kingdom); collagen XII (Santa Cruz Biotechnology, Dallas, Texas, USA); Sec16A (Sigma-Aldrich); rat hemagglutinin (Roche) or mouse hemagglutinin (Santa Cruz Biotechnology, Dallas, Texas, USA).

\section{Acknowledgements}

We would like to thank the family for their participation.

\section{Additional information}

Competing interests

Vivek Malhotra: VM is Senior Editor of Elife. The other authors declare that no competing interests exist.

Funding

\begin{tabular}{lll} 
Funder & Grant reference number & Author \\
\hline $\begin{array}{l}\text { Ministerio de Economía y } \\
\text { Competitividad }\end{array}$ & SEV-2012-0208 & $\begin{array}{l}\text { Ombretta Foresti } \\
\text { Ishier Raote } \\
\text { Vivek Malhotra }\end{array}$ \\
$\begin{array}{lll}\text { Ministerio de Economía y } \\
\text { Competitividad }\end{array}$ & BFU2013-44188-P & $\begin{array}{l}\text { Ombretta Foresti } \\
\text { Ishier Raote } \\
\text { Vivek Malhotra }\end{array}$ \\
\hline $\begin{array}{l}\text { Ministerio de Economía y } \\
\text { Competitividad }\end{array}$ & CSD2009-00016 & $\begin{array}{l}\text { Ombretta Foresti } \\
\text { Ishier Raote } \\
\text { Vivek Malhotra }\end{array}$ \\
\hline $\begin{array}{l}\text { Ministerio de Economía y } \\
\text { Competitividad }\end{array}$ & IJCl-2017-34751 & Ishier Raote \\
\hline $\begin{array}{l}\text { Ministerio de Economía y } \\
\text { Competitividad }\end{array}$ & RYC-2016-20919 & Ombretta Foresti
\end{tabular}

The funders had no role in study design, data collection and interpretation, or the decision to submit the work for publication.

Author contributions

Caroline Lekszas, Validation, Investigation, Visualization, Methodology, Writing - original draft; Ombretta Foresti, Validation, Investigation, Visualization, Methodology, Writing - review and editing; Ishier Raote, Supervision, Validation, Investigation, Visualization, Methodology, Writing - review and editing; Daniel Liedtke, Eva-Maria König, Barbara Vona, Supervision, Methodology; Indrajit Nanda, Supervision, Investigation; Peter De Coster, Rita Cauwels, Resources, Investigation; Vivek Malhotra, Thomas Haaf, Conceptualization, Supervision, Project administration, Writing - review and editing

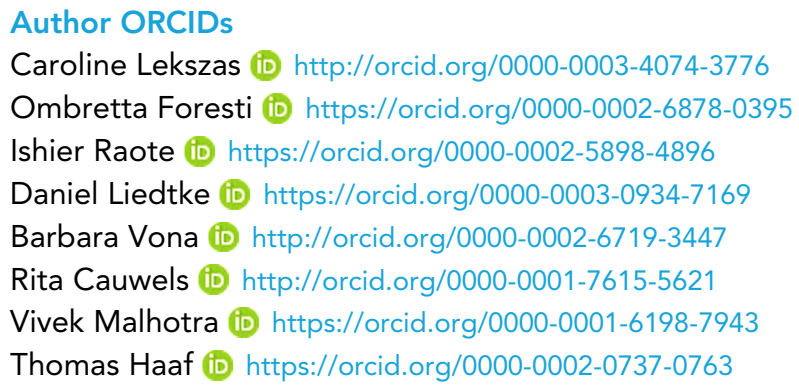


Ethics

Human subjects: Informed consent from affected individuals and/or their parents was obtained prior to initiating our investigation. Consent for publication of clinical data and genetic testing results was obtained from the affected individuals and/or their parents. This study was approved (205/11 and 46/15) by the Ethics Committee of University of Würzburg and was performed in accordance with the Declaration of Helsinki.

Decision letter and Author response

Decision letter https://doi.org/10.7554/eLife.51319.sa1

Author response https://doi.org/10.7554/eLife.51319.sa2

\section{Additional files}

Supplementary files

- Supplementary file 1. Whole Exon Sequencing (WES) was performed in the four affected bothers and their parents. Shown are the 10 variants found to be homozygous in all affected children and heterozygous in both parents.

- Transparent reporting form

Data availability

All data generated or analysed during this study are included in the manuscript and supporting files.

\section{References}

Bard F, Casano L, Mallabiabarrena A, Wallace E, Saito K, Kitayama H, Guizzunti G, Hu Y, Wendler F, Dasgupta R, Perrimon N, Malhotra V. 2006. Functional genomics reveals genes involved in protein secretion and golgi organization. Nature 439:604-607. DOI: https://doi.org/10.1038/nature04377, PMID: 16452979

Bosserhoff AK, Moser M, Schölmerich J, Buettner R, Hellerbrand C. 2003. Specific expression and regulation of the new melanoma inhibitory activity-related gene MIA2 in hepatocytes. Journal of Biological Chemistry 278. 15225-15231. DOI: https://doi.org/10.1074/jbc.M212639200, PMID: 12586826

Burn TC, Connors TD, Klinger KW, Landes GM. 1995. Increased exon-trapping efficiency through modifications to the pSPL3 splicing vector. Gene 161:183-187. DOI: https://doi.org/10.1016/0378-1119(95)00223-S, PMID: 7665076

Calfon M, Zeng H, Urano F, Till JH, Hubbard SR, Harding HP, Clark SG, Ron D. 2002. IRE1 couples endoplasmic reticulum load to secretory capacity by processing the XBP-1 mRNA. Nature 415:92-96. DOI: https://doi.org/ 10.1038/415092a, PMID: 11780124

Cauwels RG, De Coster PJ, Mortier GR, Marks LA, Martens LC. 2005. Dentinogenesis imperfecta associated with short stature, hearing loss and mental retardation: a new syndrome with autosomal recessive inheritance? Journal of Oral Pathology and Medicine 34:444-446. DOl: https://doi.org/10.1111/j.1600-0714.2005.00318.x, PMID: 16011615

Deshmukh SN, Dive AM, Moharil R, Munde P. 2016. Enigmatic insight into collagen. Journal of Oral and Maxillofacial Pathology 20:276. DOI: https://doi.org/10.4103/0973-029X.185932, PMID: 27601823

Fan J, Wang Y, Liu L, Zhang H, Zhang F, Shi L, Yu M, Gao F, Xu Z. 2017. cTAGE5 deletion in pancreatic $\beta$ cells impairs proinsulin trafficking and insulin biogenesis in mice. The Journal of Cell Biology 216:4153-4164. DOI: https://doi.org/10.1083/jcb.201705027, PMID: 29133483

Ishikawa Y, Ito S, Nagata K, Sakai LY, Bächinger HP. 2016. Intracellular mechanisms of molecular recognition and sorting for transport of large extracellular matrix molecules. PNAS 113:E6036-E6044. DOI: https://doi.org/10. 1073/pnas.1609571113, PMID: 27679847

Kang T, Boland BB, Alarcon C, Grimsby JS, Rhodes CJ, Larsen MR. 2019. Proteomic analysis of restored insulin production and trafficking in obese diabetic mouse pancreatic islets following euglycemia. Journal of Proteome Research 18:3245-3258. DOI: https://doi.org/10.1021/acs.jproteome.9b00160, PMID: 31317746

Kim J-W, Hu JC-C, Lee J-I, Moon S-K, Kim Y-J, Jang K-T, Lee S-H, Kim C-C, Hahn S-H, Simmer JP. 2005. Mutational hot spot in the DSPP gene causing dentinogenesis imperfecta type II. Human Genetics 116:186191. DOI: https://doi.org/10.1007/s00439-004-1223-6

Lek M, Karczewski KJ, Minikel EV, Samocha KE, Banks E, Fennell T, O’Donnell-Luria AH, Ware JS, Hill AJ, Cummings BB, Tukiainen T, Birnbaum DP, Kosmicki JA, Duncan LE, Estrada K, Zhao F, Zou J, Pierce-Hoffman E, Berghout J, Cooper DN, et al. 2016. Analysis of protein-coding genetic variation in 60,706 humans. Nature 536:285-291. DOI: https://doi.org/10.1038/nature19057, PMID: 27535533 
Maeda M, Saito K, Katada T. 2016. Distinct isoform-specific complexes of TANGO1 cooperatively facilitate collagen secretion from the endoplasmic reticulum. Molecular Biology of the Cell 27:2688-2696. DOI: https:// doi.org/10.1091/mbc.e16-03-0196, PMID: 27413011

Malhotra V, Erlmann P. 2011. Protein export at the ER: loading big collagens into COPII carriers. The EMBO Journal 30:3475-3480. DOI: https://doi.org/10.1038/emboj.2011.255, PMID: 21878990

Malhotra V, Erlmann P. 2015. The pathway of collagen secretion. Annual Review of Cell and Developmental Biology 31:109-124. DOI: https://doi.org/10.1146/annurev-cellbio-100913-013002, PMID: 26422332

Miller EA, Schekman R. 2013. COPII - a flexible vesicle formation system. Current Opinion in Cell Biology 25 : 420-427. DOI: https://doi.org/10.1016/j.ceb.2013.04.005, PMID: 23702145

Nogueira C, Erlmann P, Villeneuve J, Santos AJ, Martínez-Alonso E, Martínez-Menárguez JÁ, Malhotra V. 2014. SLY1 and syntaxin 18 specify a distinct pathway for procollagen VII export from the endoplasmic reticulum. eLife 3:e02784. DOI: https://doi.org/10.7554/eLife.02784, PMID: 24842878

Raote I, Ortega Bellido M, Pirozzi M, Zhang C, Melville D, Parashuraman S, Zimmermann T, Malhotra V. 2017. TANGO1 assembles into rings around COPII coats at ER exit sites. The Journal of Cell Biology 216:901-909. DOI: https://doi.org/10.1083/jcb.201608080, PMID: 28280121

Raote I, Ortega-Bellido M, Santos AJ, Foresti O, Zhang C, Garcia-Parajo MF, Campelo F, Malhotra V. 2018. TANGO1 builds a machine for collagen export by recruiting and spatially organizing COPII, tethers and membranes. eLife 7:e32723. DOI: https://doi.org/10.7554/eLife.32723, PMID: 29513218

Raote I, Malhotra V. 2019. Protein transport by vesicles and tunnels. Journal of Cell Biology 218:737-739. DOI: https://doi.org/10.1083/jcb.201811073, PMID: 30718263

Reynolds HM, Zhang L, Tran DT, Ten Hagen KG. 2019. Tango1 coordinates the formation of endoplasmic reticulum/Golgi docking sites to mediate secretory granule formation. Journal of Biological Chemistry 294 19498-19510. DOI: https://doi.org/10.1074/jbc.RA119.011063, PMID: 31690624

Saito K, Chen M, Bard F, Chen S, Zhou H, Woodley D, Polischuk R, Schekman R, Malhotra V. 2009. TANGO1 facilitates cargo loading at endoplasmic reticulum exit sites. Cell 136:891-902. DOl: https://doi.org/10.1016/j. cell.2008.12.025

Saito K, Yamashiro K, Ichikawa Y, Erlmann P, Kontani K, Malhotra V, Katada T. 2011. cTAGE5 mediates collagen secretion through interaction with TANGO1 at endoplasmic reticulum exit sites. Molecular Biology of the Cell 22:2301-2308. DOI: https://doi.org/10.1091/mbc.e11-02-0143, PMID: 21525241

Santos AJ, Raote I, Scarpa M, Brouwers N, Malhotra V. 2015. TANGO1 recruits ERGIC membranes to the endoplasmic reticulum for procollagen export. eLife 4:e10982. DOI: https://doi.org/10.7554/eLife.10982, PMID: 26568311

Santos AJ, Nogueira C, Ortega-Bellido M, Malhotra V. 2016. TANGO1 and Mia2/cTAGE5 (TALI) cooperate to export bulky pre-chylomicrons/VLDLs from the endoplasmic reticulum. The Journal of Cell Biology 213:343354. DOI: https://doi.org/10.1083/jcb.201603072, PMID: 27138255

Seelow D, Schuelke M, Hildebrandt F, Nürnberg P. 2009. HomozygosityMapper-an interactive approach to homozygosity mapping. Nucleic Acids Research 37:W593-W599. DOI: https://doi.org/10.1093/nar/gkp369, PMID: 19465395

Wilson DG, Phamluong K, Li L, Sun M, Cao TC, Liu PS, Modrusan Z, Sandoval WN, Rangell L, Carano RA, Peterson AS, Solloway MJ. 2011. Global defects in collagen secretion in a Mia3/TANGO1 knockout mouse. The Journal of Cell Biology 193:935-951. DOI: https://doi.org/10.1083/jcb.201007162, PMID: 21606205

Xiao S, Yu C, Chou X, Yuan W, Wang Y, Bu L, Fu G, Qian M, Yang J, Shi Y, Hu L, Han B, Wang Z, Huang W, Liu J, Chen Z, Zhao G, Kong X. 2001. Dentinogenesis imperfecta 1 with or without progressive hearing loss is associated with distinct mutations in DSPP. Nature Genetics 27:201-204. DOI: https://doi.org/10.1038/84848, PMID: 11175790

Ye J, Coulouris G, Zaretskaya I, Cutcutache I, Rozen S, Madden TL. 2012. Primer-BLAST: a tool to design targetspecific primers for polymerase chain reaction. BMC Bioinformatics 13:134. DOI: https://doi.org/10.1186/1471 2105-13-134, PMID: 22708584 